\title{
分叉问题的几何描述及其计算方法 ${ }^{*}$
}

\author{
季海波 武际可 胡海昌 \\ (北京大学力学系, 北京 1000871)
}

\section{摘 要}

本文通过一个构造的向量场, 建立了非线性代数方程组的解流形与对应向量场 的不变流形之间的等价关系, 把解流形的分又问题转化为对向量场奇点附近局部性 态的研究. 这种思路具有几何直观性, 并为解流形的跟踪与分叉计算提供了一套新 的数值方法.

\section{关键词：非线性,分又计算}

\section{一、引 言}

考查如下的带参数的非线性方程组

$$
F(x, \lambda)=0,
$$

式中 $F: R^{n} \times R \rightarrow R^{n}$.

许多实际问题, 尤其是物理系统的平衡状态分析, 当需要数值求解的时候, 一般要化成形 如方程 (1.1)的有限维非线性方程组. 当前, 线性系统的求解已经日趋成熟，但对于非线性问 题还存在着实质性困难, 其中包括解的多重性、存在转折点、分叉点之类的奇异性现象, 而这些 奇异点附近往往对应着物理状态的一种突变或跳跃，所以关于奇异性问题的数值方法引起了 人们的兴趣和重视 ${ }^{[1-3]}$.

这里限定方程 (1.1) 是单参数系统, 救数 $F$ 是连续可微的, 这样一来一般地设方程 (1.1)的 解定义了 $R^{n+1}$ 空间中的曲线。为了了解当参数连续变化时,方程 (1.1)之解的发展过程, 带有 预测-修正过程的延续算法常常被用来跟踪解曲线。文献 [4]提出的拟弧长方法改进了以前的 延续算法, 并希望从 $\operatorname{det}\left(D_{x} F\right)$ 的符号变化及时探明解的分叉情况. 文献 $[4,5]$ 各自提出了 不同的分叉方程可用来求解分叉方问，他们的手法大体上是分析的，似乎还难以形成一个系 统的成功率较高的分叉问题的计算方法.

本文将运用向量场、流形等几何观点, 把非线性方程 (1.1)的解流形与相应的向量场之不 变流形联系起来, 使延续算法及其它问题的表述更为简洁、自然. 我们将利用向量场的旋转度 的工具来判断分叉点的存在性, 从向量场在奇点附近的局部性态得出新的计算分叉方向的公 式,并在多重分叉的情形引进了单纯形计算技术. 文中最后给出了一个流体力学的计算实例.

本文1990年 7 月 3 日收到, 1991 年 1 月 11 泊收到修改稿.

*本文系博士点墓会资助项目. 


\section{二、能流形与不变流形}

记 $y=(x, \lambda)$,忽略状态变量 $x$ 与参变量 $\lambda$ 的差别, 可把方程 (1.1) 改写成

$$
F(y)=0 \text {, }
$$

这里 $F=\left(F^{1}, \cdots, F^{n}\right)^{T} \in R^{n}$, 而 $y=\left(y^{1}, \cdots, y^{n+1}\right)^{T} \in R^{n+1}$.

我们总是假定函数 $F$ 是足够光滑的, 一般地讲方程 (2.1) 的解除个别点外淟个一维流形, 我们称之为解流形. 函数 $F$ 的 Fréchet 导数 $D F(y)=\left(\begin{array}{c}\partial F^{\prime} \\ c y^{j}\end{array}\right)$ 是个 $n \times(n+1)$ 矩阵, 它 也是光滑的。

现在定义一个 $R^{n+1}$ 上的向量场

$$
v^{\prime}=\left(v^{1}, \cdots, v^{n+1}\right)^{T},
$$

它的每个分量 $\eta^{i}$ 系由 $D F$ 的第 $j$ 列之余因子构成, 即

$$
\begin{aligned}
& v^{\prime}=(-1)^{\prime} \operatorname{det}\left(\frac{\partial F}{\partial y^{i}}, \cdots, \frac{\partial F}{\partial y^{j-1}}, \frac{\widehat{\partial F}}{\partial y^{j}}, \frac{\partial F}{\partial y^{j+1}}, \cdots \frac{\partial F}{\partial y^{n+1}}\right), \\
& j=1, \cdots, n+1 ;
\end{aligned}
$$

式中符号“人”表示去掉所指之列。

这个向量场是光滑的, 且具有性质

$$
D F \cdot \nu(y)=0, \forall y \in R^{n+1} \text {; }
$$

该式可从等式

$$
\operatorname{det}\left(\begin{array}{c}
D F \\
\frac{\partial F^{i}}{\partial y^{1}}, \cdots, \frac{\partial F^{i}}{\partial y^{n+1}}
\end{array}\right)=0, i-1, \cdots, n_{3},
$$

左边行列式按最后一行展开得到.

向量场 $v$ 伴随着一个动力系统

$$
\frac{d y}{d t}=t^{\prime}(y)
$$

定义 2.1. 称 $y$ 是向量场 $v$ 的正则点系指 $\nu(y) \neq 0 \in R^{n+1}$; 反之若 $\nu(y)=0$ 则称 $y$ 是 向量场 $v$ 的奇异点.

定义 2.2. 一个流形 $M \subset R^{n+1}$ 称为向量场 $\nu$ 的不变流形, 是指 $\forall y \in M$, 在系统(2.5)决 定的流动之下,它仍保持在 $M$ 上.

命题 2.1. $F(y)=$ const 的解流形(或称 $F$ 的等值流形) 是问量场 $\nu$ 的不变流形.

命题 2.2. 如果向量场 $v$ 的一个一维不变流形上或者没有奇点、或者只包含在其上. 为孤 立的奇点, 则该不变流形亦为 $F(y)=\mathrm{const}$ 的解流形.

考虑到关系 (2.3), 命题的成立是明显的, 这里略去证明过程. 说明一点, 我们限定命题 2.2 中的不变流形是一维的且其上只能有孤立奇点, 是为了使不变流形上函数 $F$ 之值等于相同的 常数.

这样一来我们了解到, $F$ 的等值流形与诱导向量场 $v$ 的不变流形之间的紧密联系, 而后者 提供了一个直观的几何图景. 特别我们要解方程 (2.1)即求 $F$ 的零值流形, 便转化为求向量场 
$v$ 经过某点的积分曲线。

\section{三、解流形的分叉}

由隐函数定理容易知道解流形上的分叉点(即两个或多个解流形的交叉点)一定是诱导向 量场 $v$ 的奇点, 所以我们可以先搜索解流形上的奇点, 然后再来确认它是否为尔叉点并求出过 该点的分叉方向.

在延续跟踪解流形的实际计算过程中, 我们要求根据奇异点的開围环境来判断奇点的存 在性. 显然解流形上向量 $v$ 的变号是奇点存在的一个简单情形, 这里我 们将利用问量场的旋转度的工具给出一个较为一般的判断条件.

设 $R^{n+1}$ 中 $n$ 维闭的超曲面 $S$ 有保持定向的局部坐标 $u^{1}, \cdots, u^{n}$; 且 唀导向最场 $v$ 在 $S$ 上没有奇点, 则向量场 $v$ 在 $S$ 上的旋转度可表示为

$$
\operatorname{deg}_{s} v=\frac{1}{\gamma_{n}} \int_{s} \frac{1}{\|v\|^{n+1}} \operatorname{det}\left(\nu, \frac{\partial \nu}{\partial u^{1}}, \cdots, \frac{\partial v}{\partial u^{n}}\right) d u^{1} \wedge \cdots \wedge a^{\prime} u^{n},
$$

式中 $r_{n}=2 x^{\frac{n+1}{2}} / \Gamma\left(\frac{n+1}{2}\right)$ 是 $n$ 维单位球面的体积, $\Gamma(\cdot)$ 是 Gamma 特殊函数, 䜣・为欧氏模.

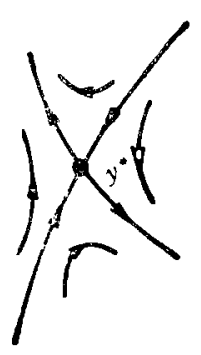

图 1 奇点附近 的不变流形

在几何上 (3.1) 式里的积分意为 $S$ 上的问量场 $\nu$ 之方向所张的空间角（按照一定的定向）. 旋转度 $\operatorname{deg}_{s} \boldsymbol{v}$ 总是取整数值, 它的一个基本性质是如果浰曲面 $S$ 内部没有奇点, 则向量场 $\nu$ 在 $S$ 上的度是零. 这就给出了判断解流形上奇点存在的一个准则.

现设 $y^{*}$ 是解流形上的一个奇点, 我们来讨论如何确定通过点 $y^{*}$ 的分叉方茼(即解流形 在奇点的切线方向). 已经知道 (2.1)式的解流形与向量场的一类不变流形的等同关系, 一个 自然的想法就是通过考查向量场 $v$ 在奇点 $y^{*}$ 附近的轨道走向(图 1)来了解分叉方向.

因为 $\nu\left(y^{*}\right)=0, \mathrm{Jacobi}$ 矩阵 $D F^{*}=D F\left(y^{*}\right)$ 是一个退化的 $n \times(n+1)$ 矩阵, 从而 $D F^{*}$ 的零空间可以莅成

$$
\mathscr{N}\left(D F^{*}\right)=\operatorname{span}\left\{\phi_{1}, \cdots, \phi_{m}\right\} \subset R^{n+1},
$$

式中 $\phi_{1}, \cdots, \phi_{m}$ 是单位正交的们量组.

通”陪 $\mathscr{N}\left(D F^{*}\right)$ 的维数 $m$ 远小于实际问题的维数 $n$, 容易证明任何在 $y^{*}$ 分叉的方向 $d$ 都在子空间 $\mathscr{N}\left(D F^{*}\right)$ 内, 于是

$$
d=\sum_{i=1}^{m} \xi^{t} \phi_{i}=\Phi \xi,
$$

这里 $\Phi=\left(\phi_{1}, \cdots, \phi_{m}\right)$ 是个 $(n+1) \times m$ 知阵, 而 $\xi=\left(\xi^{1}, \cdots, \xi^{m}\right)^{T} \in R^{m}$.

定义一个 $R^{m}$ 上的缩减问量场

$$
p(\xi)=\psi^{T} \cdot \nu\left(y^{*}+\varphi \xi\right) \text {. }
$$

团为分叉方向限制在子空间 $\mathscr{N}\left(D F^{*}\right)$ 内, 我们希望向量场 $p(\xi)$ 经过奇点 $\xi=0$ 的朁部不 变流形对应着分叉方向。

Hartman-Grobman 定理 ${ }^{[6]}$ 指出, 向量场的双曲型奇点附近的局部性态与在该点的线性化 泃量场的局部性态是拓扑等价的, 并且两者经过奇点的不变流形在奇点处相切. 据此我们得 到 
定理 3.1. 如果线性特征值问题

$$
A \xi=\mu \xi, A=\stackrel{\text { def }}{=} D p(0)=\Phi^{T} \cdot D v\left(y^{*}\right) \cdot \Phi,
$$

有 $m$ 个相异的非零实特征值, 则其对应的各个特征方向按 (3.3)式对应到分叉方向.

这个结果是简洁明了的, 但是该方法只能在 $m=2$, 且为简单分叉的情形下有效. 因为 当 $m>2$ 时, $\operatorname{Rank}\left(D F^{*}\right)=n+1-m<n-1, D F^{*}$ 的所有 $(n-1) \times(n-1)$ 子矩阵 的行列式均为零, 根据 (2.2) 式向量场 $v$ 的构造法, 这将使得 $D v\left(y^{*}\right)$ 的所有元素均为零 (事实 上向量场 $v$ 在 $y^{*}$ 的至少直到 $m-2$ 阶的 Fréchet 导数均为零), 从而 $A$ 的所有特征值都为零, 不符合定理 3.1 的条件,亦即这种线性化方法失败.

在多重分叉的情形 $(m>2)$, 我们可以根据向量场在奇点的极限方向来确定分叉方向 设 $\xi=0$ 是问量场 $p(\xi)$ 的孤立奇点, 称 $\eta \in R^{m}(\|\eta\|=1)$ 是 $p(\xi)$ 在 $\xi=0$ 的一个极限方 向是指下面两个等式:

$$
\lim _{i \rightarrow 0^{+}} \frac{p(i \eta)}{\|p(t \eta)\|}= \pm \eta
$$

其中之一成立,式中 $t \in R$.

当单位化的向量场在原奇点的极限与路径的曲率无关时, 分叉方向就是极限方向, 因此从 (3.6)式可求出分叉方向. 为了使用的方便, 我们给出 (3.6)式的另一个等价的表示, 定义(假设 存在)

$$
Q(\eta)=\lim _{i \rightarrow 0^{+}} \frac{p(t \eta)}{\eta^{T} \cdot p(t \eta)},
$$

式中 $\eta \in R^{m}$ 且 $\|\eta\|=1$, 则 $Q$ 的不动点(满足 $Q(\eta)=\eta$ )按(3.3)式给出可能的分叉方向.

\section{四、数 值方 法}

为了了解 (2.1)式的解流形的全貌, 我们通常从一个初始点开始进行延续计算一步步地跟 踪解流形. 很多情况下事先可以知道解流形上的一个初始点 $y_{0}$, 如果这样的 $y_{0}$ 末知, 或者希 望求出另一支不相交的解流形, 我们总可构造一个同伦过程来求出一个初始点 $y_{0}\left(\right.$ 设 $v\left(y_{0}\right) \neq$ 0 ).

记 $\tau(y)=v(y) /\|v(y)\|$, 通过诱导向量场可从上步点 $y_{k-1}$ 按方向 $\tau\left(y_{k-1}\right)$ 得到一个 Euler 预测 $y_{k}^{(0)}$,

$$
y_{k}^{(0)}=y_{k-1}+\tau\left(y_{k-1}\right) \cdot \Delta s_{k-1}, k=1,2, \cdots,
$$

式中 $\Delta s_{k-1}$ 是步长.

这里不需要用其它更高阶的公式, 因为每前进若干步后可以作 Newton 型迭代修正, 迭 代方向与 $v\left(y_{k}^{(0)}\right)$ 垂直, 比如

$$
y_{k}^{(l)}=y_{k}^{(l-1)}-\left(\begin{array}{c}
D F\left(y_{k}^{(0)}\right) \\
\nu^{T}\left(y_{k}^{(0)}\right)
\end{array}\right)^{-1} \cdot\left(\begin{array}{c}
F\left(y_{k}^{(l-1)}\right) \\
0
\end{array}\right), l=1,2, \cdots,
$$

只要 $\left\|v\left(y_{k}^{(0)}\right)\right\|$ 不是很小, $\boldsymbol{y}_{k}^{(0)}$ 离解流形不远, 可以指望 (4.2) 式形成的迭代序列 $y_{k}^{(1)}, \boldsymbol{y}_{k}^{(2)}, \cdots$ 能够 收玫到解流形上一点 $y_{k}$ 满足 $F\left(y_{k}\right)=0$, 迭代序列的有限步结果 $y_{k}^{(l)}$ 称为 $y_{k}^{(0)}$ 的一个修正.

(4.1)与 (4.2)式的联合运用(图 2), 形成了预测一修正的延续算法. 为了在延续计算过程 中找到解流形上可能存在的奇点, 我们应用向量场的度的办法, 为此先给出 (3.1)式的实用计 
算公式.

为简单起见, 假定 $n$ 维闭曲面 $S$ 所包含的区域是星形的, 其中心是 $y_{c}$ （即对 $S$ 内部任一 点 $y$,从 $y_{c}$ 到 $y$ 的直线段都在 $S$ 内部). 我们用 $N$ 个单纯形 $\sigma_{i}=\left\langle y_{1}^{(i)}, y_{2}^{(i)}, \cdots, y_{n+1}^{(i)}\right\rangle$, 其中 $1 \leqslant i \leqslant N$ 组成的 $n$ 维单纯复形 $\bigcup_{i=1}^{N} \sigma_{i}$ 来逼近 $S$, 记

$$
\begin{gathered}
\alpha_{i}=\operatorname{det}\left(y_{i}^{(i)}-y_{c}, y_{2}^{(i)}-y_{c}, \cdots, y_{n+1}^{(i)}-y_{c}\right), \\
\beta_{i}=\operatorname{det}\left(\tau_{1}^{(i)}, \tau_{2}^{(i)}, \cdots, \tau_{n+1}^{(i)}\right),
\end{gathered}
$$

式中 $\tau_{i}^{(i)} \stackrel{\text { def }}{=} \tau\left(y_{i}^{(i)}\right), 1 \leqslant j \leqslant n+1$; 根据 (3.1)式以及单纯部分的定向, 向量场 $v$ 在 $S$ 上的旋 转度有近似公式

$$
\operatorname{deg}_{s} v \approx \frac{1}{n ! \gamma_{n}} \sum_{i=1}^{N} \operatorname{sign}\left(\alpha_{i}\right) \cdot \beta_{i},
$$

式中 sign 是符号函数。

显然 $S$ 的形状可取得尽量简单, 因为旋转度与 $S$ 的形状无关, 只要 $S$ 内包含的奇点不变. 实际计算中还可按如下的办法只计算二维的旋转度.

设有解流形上相邻两点 $y_{0}, y_{1}$ 上的诱导向量分别为 $\nu_{0}, v_{1}$ (图 2), 定义一个平面 $E^{2}=$ $\operatorname{span}\left\{b_{1}, b_{2}\right\} \subset R^{n+1}$ ，其中 $b_{1}=v_{1} /\left\|v_{1}\right\|$ 而 $b_{2}$ 是垂直于 $b_{1}$ 的单位问量; 当 $v_{0}$ 与 $v_{1}$ 不平行 时, $b_{2}$ 在由 $v_{0}$ 与 $v_{1}$ 张成的平面内是唯一的.

作向量场 $v$ 在子平面 $E^{2}$ 中的投影向量场

$$
f(u)=\left(b_{1}^{T} \cdot v(y), b_{2}^{T} \cdot v(y)\right)^{T},
$$

式中 $y=y_{1}+u^{2} b_{1}+u^{2} b_{2}, u=\left(u^{1}, u^{2}\right)^{T} \in R^{2}$.

为了寻找 $y_{1}$ 附近解流形上可能存在的奇点, 我们可以把捜索区域限制在 $y_{1}$ 附近一个小 的闭曲线 $\Gamma \subset E^{2}$ 内, 希望向量场 $v(y)$ 在 $y_{1}$ 附近的奇点对应 着向量场 $f(u)$ 在 $\Gamma$ 内的近似奇点.

定义单位向量场 $\tau(u)=f(u) /\|f(u)\|$ 和一个定向行列式

$$
O_{i}=\operatorname{det}\left(\begin{array}{l}
f^{1}\left(c_{i}\right), f^{1}\left(c_{i+1}\right) \\
f^{2}\left(c_{i}\right), f^{2}\left(c_{i+1}\right)
\end{array}\right), i=1, \cdots, N,
$$

这里 $c_{1}, \cdots, c_{N}$ 是 $\Gamma$ 上按逆时针方向排序的一组点 (图 2)且 $c_{N+1}=c_{1}$, 而 $O_{i}>0(<0)$ 便表示 $\left\{f\left(c_{i}\right), f\left(c_{i+1}\right)\right\}$ 的定向与 原坐标系定向 $\left\{\left(\begin{array}{l}1 \\ 0\end{array}\right),\left(\begin{array}{l}0 \\ 1\end{array}\right)\right\}$ 相同(相反).

考虑到当 $O_{i} \approx 0$ 时将不好定向，实际上我们是计算 $f$ 的 扰动向量场子的旋转度并用公式

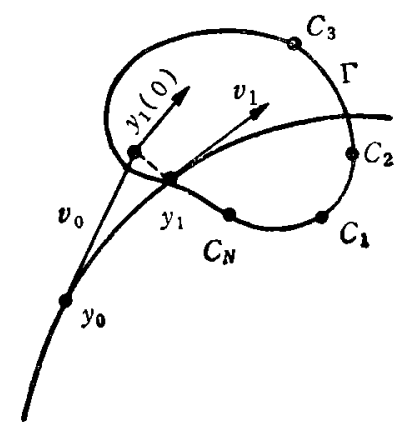

图 2 解流形的跟踪与奇点判断

$$
\left.\operatorname{deg}_{I}\right\}=-\frac{1}{2 \pi} \sum_{i=1}^{N} S_{i} \cdot \arccos \left(\tau_{i}^{T} \cdot \tau_{i+1}\right),
$$

其中 $\tau_{i}-\tau\left(c_{i}\right)$ 对于 $1 \leqslant i \leqslant N$ 和 $\tau_{N+1}=\tau_{1}$; 并且

$$
S_{i}=\left\{\begin{array}{ll}
+1, & O_{i}>\varepsilon \\
-1, & O_{i}<-\varepsilon \\
\pm 1 \text { (不定) }, & -\varepsilon \leqslant O_{i} \leqslant \varepsilon
\end{array}, i=1, \cdots, N,\right.
$$


式中 $\varepsilon$ 是适当选择的小正数.

对于 $S_{i}$ 的不定部分的任意正负组合, (4.7a) 式均给出扰动向量场 $f$ 可能的旋转度, 如果 其中之一是非零整数则意味着 $\Gamma$ 内可能存在奇点. 一旦 $\operatorname{deg}_{T} f$ 表明 $\Gamma$ 内存在奇点, 我们可以 在 $\Gamma$ 内进行分割计算旋转度直到求出满足一定精度要求的奇点为止.

这个办法可以与延续算法相结合, 在追踪解流形的过程中不时地计算局部范围的旋转度 来判断前方可能存在的奇点, 该法可避免在奇点附近进行类似于 (4.2)式的牛顿迭代, 因而成 功率较高. 一个值得推荐的方式是在解流形上把搜索区域取成一系列等边三角形, 整个计算 过程顶着三角形朝前走.

如果找到了解流形上一个奇点 $y^{*}$, 为了计算经过 $y^{*}$ 的分叉方向, 首先要求出 $D F^{*}$ 的 零空间的单位正交向量组 $\phi_{1}, \cdots, \phi_{m}$.

在简单分叉的情形 $(m=2)$, 求分叉方向化为线性特征值问题 (3.5), 其中矩阵 $A$ 可写成 方向异数的形式

$$
A=\left(\phi_{i}^{T} \frac{\partial v}{\partial \phi_{i}}\right),
$$

数值计算时为了避免求向量场 $v$ 的微分, 可用如下近似公式:

$$
A \approx \frac{1}{\varepsilon}\left(\begin{array}{l}
\phi_{1}^{T} \cdot v\left(y^{*}+\varepsilon \phi_{1}\right), \phi_{1}^{T} \cdot \nu\left(y^{*}+\varepsilon \phi_{2}\right) \\
\phi_{2}^{T} \cdot v\left(y^{*}+\varepsilon \phi_{1}\right), \phi_{2}^{T} \cdot v\left(y^{*}+\varepsilon \phi_{2}\right)
\end{array}\right),
$$

其中 $\varepsilon$ 是小正数,它的特征方向 $\xi=\left(\xi^{1}, \xi^{2}\right)^{T}$ 确定了近似分叉方向 $d-\xi^{1} \phi_{1}+\xi^{2} \phi_{2}$.

对于多重分叉,需求解向量场的极限方向, 取小正数 $\varepsilon$, 可把 (3.7)式之 $Q(\eta)$ 的不动点 方程化为有限形式

式中 $\|\eta\|=1$.

$$
q(\eta)=\stackrel{\text { def }}{=} p(\varepsilon \eta)-\left(\eta^{T} \cdot p(\varepsilon \eta)\right) \eta=0,
$$

可以看出, 向量场 $q(\eta)$ 定义在单位球面 $S^{m-1}=\left\{\eta \in R^{m} \mid\|\boldsymbol{\eta}\|-1\right\}$ 上并与 $S^{\boldsymbol{m}^{-1}}$ 相切, 等式 (4.9)在几何上是直观的, 它相当于求球面向量场 $q(\eta)$ 的奇点.

我们用单纯形法求解 (4.9) 式, 因解流形是光滑的, 只需对球面 $S^{m-1}$ 的一半 $\left\{\eta=\left(n^{1}, \cdots\right.\right.$, $\left.\left.\eta^{m}\right)^{T} \in S^{m-1} \mid \eta^{m} \geqslant 0\right\}$ 作单纯部分逼近, 它的部分椝则可从单位立方块表面的单纯部分通过径 向映射得来.

考查某个单纯形 $\sigma=\left\langle\eta_{1}, \eta_{2}, \cdots, \eta_{m}\right\rangle$,作 (4.9)式在该单形上的投影方程

$$
q_{\sigma}(\xi)=\stackrel{\operatorname{det}}{-}\left(\eta_{1}-\eta_{m}, \eta_{:}-\eta_{m}, \cdots, \eta_{m-1}-\eta_{m}\right)^{T} \cdot q(\xi)=0,
$$

这里 $q_{\sigma}: \xi \in \sigma \longmapsto R^{m-1}$, 只姴单形 $\sigma$ 是小的, $q(\xi)$ 将与 $\sigma$ 近似平行, 因此只需求 $q_{\sigma}(\xi)$ 的零 点就可以了.

可以证明 $[7]$ 如果方程

$$
\left(\begin{array}{cccc}
q_{\sigma}\left(\eta_{1}\right), & q_{\sigma}\left(\eta_{2}\right), & \cdots, & q_{,}\left(\eta_{m}\right) \\
1, & 1, & \cdots, & 1
\end{array}\right)\left(\begin{array}{c}
w^{1} \\
w^{2} \\
\vdots \\
w^{m}
\end{array}\right)=\left(\begin{array}{c}
0 \\
\vdots \\
0 \\
1
\end{array}\right)
$$

有解 $w^{i} \geqslant-\delta, 1 \leqslant i \leqslant m$ (为了防止数值误差, 又引人了小正数 $\delta$ ), 则 (4.10)式便有近似 
解 $\xi=\left(\xi^{1}, \cdots, \xi^{m}\right)^{T}=\sum_{i=1}^{m} w^{i} \eta_{i}$, 从而得到近似分叉方向 $d=\sum_{i=1}^{m} \xi^{i} \phi_{i}$.

一般 $\mathscr{N}\left(D F^{*}\right)$ 的维数 $m$ 较小, 我们有能力对半球面上所有单形搜索一遍, 期望得到尽 可能全部的分叉方向。如找到了好几个分叉方向, 则当可任选一支使延续算法沿此继续下 去.

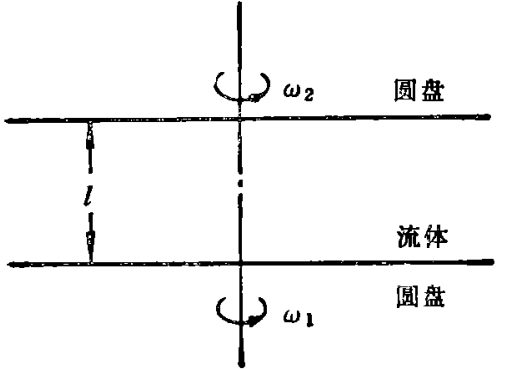

图 3 旋转圆盘流动师题

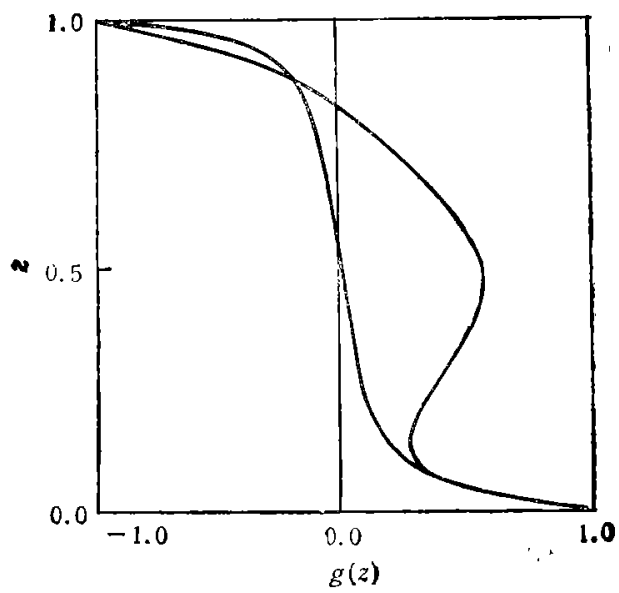

图 4 分叉后 $(\mu=-1, R e=300)$ 的环向速度分布

作为计算实例，我们求解了半径无限大的两个平行旋转圆盘之间粘性流体的定常流动（图 3),按照轴对称的假定和无量纲化的过程 ${ }^{[8]}$,流体运动方程可简化为常微分方程组

$$
\left\{\begin{array}{l}
f^{\mathrm{IV}}-R_{e} \cdot\left(f^{\prime \prime \prime} f+4 g^{\prime} g\right)=0, \\
g^{\prime \prime}+R_{e} \cdot\left(f^{\prime} g-g^{\prime}\right)=0, \\
f(0)=f^{\prime}(0)=f(1)=f(1)=0, \\
g(0)=1, g(1)=\mu,
\end{array}\right.
$$

式中 $f(z)$ 为轴向流动速度, $g(z)$ 区映了环向流动速度， $z \in[0,1], \mu=\omega_{2} / \omega_{1}$ 是上下圆 盘转速比, Reynolds 数 $R e=\omega_{1} l^{2} / \nu, l$ 为圆盘间距, $v$ 表运动粘度.

计算中对 (4.12)式采用等距差分离散方式 ${ }^{[7]}$, 在对上下圆盤转速相同方向相反 $(\mu=-$ I) 的情形计算时发现了分叉. 对于不同的离散方程数, 临界 Reynolds 数计算结梁列于表 1.

表 1 转速比 $\mu=-1$ 时流体运动的分叉点

\begin{tabular}{|c|c|c|c|}
\hline Reynolds 数 & $N=18$ & $N=38$ & $N=78$ \\
\hline$R e_{\mathrm{c}}$ & 103.118 & 114.801 & $118 . \div 77$ \\
\hline
\end{tabular}

这是个简单分叉, 文献 [8]通过其它途径得到临界 Reynolds 数 $R e_{\mathrm{i}}=119.8$, 出现分叉 后, 除了原来的对中间平行面 $z=\frac{1}{2}$ 反对称的旋转慔式之外, 还有二种上下颠倒的非对称模 
式(图 4, 只画出其中之一), 实验 ${ }^{[9]}$ 也证实了失稳过程和流体的非对称运动形式. 我们的计算 有效地实现了跟踪初始解流形, 判断并确定分叉点, 计算分叉方向, 追踪分叉后各支路径等一 系列过程.

\section{参考文 献}

[1] 武际可等,力学与实践,9(1987),1-7.

[2] 朱正佑,近代数学与力学, 北京大学出版社, 1987,235-252.

[3] Küpper, T. et al. (Eds). Numerical Method for Bifurcation Problems, ISNM70, Birkhăuser-Verlag, 1984.

[4] Keller, H.B., in Applications of Bifurcation Theory (Ed. Rabinowitz, P.H.), Academic Press, New York, 1977, 359-384.

15] Kearfott, R.B., in [3], 210-218.

[6] Hartman, P., Ordinary Differential Equations, Wile New York, 1964.

[ 7 ] 李灰扬等,非线性方程组的数值解法,科学出版社, 1987 .

[8] Brady. J.F. et al., J. Fluid Mech.. 175(1987),363-394.

[9] Szeri, A.L. et al., ibid, 134(1983), 133-154. 